\title{
Naturally Occurring 1,5-Diarylpentanoids: A Review
}

\author{
Neslihan Celebioglu ${ }^{\oplus 1}$, Ufuk Ozgen ${ }^{\oplus 2}$ and Hasan Secen ${ }^{\oplus *}$ \\ ${ }^{I}$ Ataturk University, Faculty of Sciences, Department of Chemistry, 25240 Erzurum, Türkiye \\ ${ }^{2}$ Karadeniz Technical University, Faculty of Pharmacy, Department of Pharmacognosy, \\ TR-61080 Trabzon, Türkiye
}

(Received October 17, 2017; Revised November 03, 2017; Accepted November 05, 2017)

\begin{abstract}
Diarylpentanoids, having Ar-C5-Ar chain, are a small class of natural products, most of which have been discovered in last the decade. This review comprises 20 natural diarylpentanoids, including their isolation, characterization and biological activities.
\end{abstract}

Keywords: Diarylpentanoids; natural product; biological activity. (C2017 ACG Publications. All rights reserved.

\section{Introduction}

Diarylpentanoids are analogs of diarylheptanoids ${ }^{1-7}$ found widespread in nature. So far, over 400 Ar-C7-Ar, having diarylheptanoid structure were isolated, reporting their various biological activities such as anticancer, antiemetic, antibacterial, antioxidant and anti-inflammatory. However, diarylpentanoids with Ar-C5-Ar structure are not as widespread as diarylheptanoids in natural sources; only 20 diarylpentanoid compounds have been reported to be isolated (Figure 1, Table 1). This article is the first review on diarylpentanoids, comprising their isolation, characterization and biological activities.

\section{Natural 1,5-Diarylpentanoids}

Daphneolone (1) is the most commonly found natural diarylpentanoid, posessing Ar-C5-Ar skeleton and was first isolated from the roots of Daphne odora (Thymelaeaceae) ${ }^{8}$. Its structure was elucidated using spectroscopic methods such as ${ }^{1} \mathrm{H}-\mathrm{NMR}$, UV and MS. Its melting point and specific optical rotation were determined to be $119-120^{\circ} \mathrm{C}$ and $[\alpha]_{\mathrm{D}}^{23}=+10^{\circ}(\mathrm{c} 1.1, \mathrm{MeOH})$, respectively. Later, it was isolated from the roots of Daphne tangutica, melting point and specific optical rotation of which were reported to be $118-119^{\circ} \mathrm{C}$ and $[\alpha]_{\mathrm{D}}^{23}=+9^{\circ}(c 1.01, \mathrm{MeOH})$, respectively ${ }^{9}$.

\footnotetext{
* Corresponding author: E-Mail: hsecen@atauni.edu.tr
}

The article was published by ACG Publications 
Daphneolone (1) was found in the bark of Daphne mezereum ${ }^{10}$ using HPLC technique. It was isolated from Daphne odora var. atrocaulis ${ }^{11}$ and aerial parts of Daphne pedunculata together with known seven compounds ${ }^{12}$. Compounds $(+)-1,(+)-11\left([\alpha]_{\mathrm{D}}{ }^{20}=+6.1^{\circ}, c 0.12, \mathrm{MeOH}\right)$, and $(+)-\mathbf{1 2}\left([\alpha]_{\mathrm{D}}{ }^{20}=+12.2^{\circ}, c\right.$ $0.13, \mathrm{MeOH})$ were isolated from tissue culture cells of Daphne giraldii, structures of which were elucidated using spectroscopic methods ${ }^{13}$. While $\mathbf{1}$ and $\mathbf{1 0}$ were also isolated from the stems and leaves of Daphne bholua ${ }^{14}, \mathbf{1}$ was obtained from the bark and roots of Daphne retusa ${ }^{15}$. Moreover, $(S,+)-\mathbf{1}\left([\alpha]_{\mathrm{D}}{ }^{25}=\right.$ $\left.+4.7^{\circ}, c 1.23, \mathrm{MeOH}\right), \mathbf{6}, \mathbf{9}, \mathbf{1 0}$ and 18 were isolated from Daphne acutiloba. Compounds $\mathbf{1}, \mathbf{9}$ and 18 were reported to have anti-HIV activity, among which daphnenin (18) showed the highest anti-HIV activity ${ }^{16}$. Compounds 1 and $\mathbf{9}$ were obtained from EtOAc extract of the aerial part of Thymelaea lythroides (Thymelaeaceae) ${ }^{17}$. Daphneolone (1) and daphnenone (10) were isolated from the flower buds of Daphne genkwa ${ }^{18} \mathbf{1}$ and $\mathbf{9}$, isolated from Daphne acutiloba, displayed nematicidal activity ${ }^{19}$.

The natural products $\mathbf{2}$ and $\mathbf{3}$, obtained from methanol extract of the rhizomes of Curcuma domestica (Zingiberaceae) ${ }^{20}$ and Curcuma longa ${ }^{23}$ were reported to have strong antioxidant and antiinflammatory activities ${ }^{20}$. Compound $\mathbf{3}$ was also isolated from Curcuma longa $a^{22} .2$ and $\mathbf{8}$ were isolated from the root tubers of Curcuma long $a^{21}$. Moreover, compound 3, isolated from the rhizomes of Dioscorea nipponica (Dioscoreaceae), displayed anti-neuroinflammatory effect ${ }^{24}$. Between $\mathbf{2}$ and $\mathbf{3}$ from the methanolic extract $(80 \%)$ of the rhizomes of Curcuma xanthorrhiza, 2 was reported to have inhibitory effect toward $\mathrm{NO}^{25}$.

Compounds 4 and 5 from the roots ${ }^{26,28}$ and rhizomes ${ }^{27}$ of Stellera chamaejasme (Thymelaeaceae), showed contact activity ${ }^{26}$, good level of anti-feedant activity ${ }^{26}$ and high bioactivity against aphids ${ }^{28}$. While 4, 5, 6, $(+)-14\left([\alpha]_{\mathrm{D}}{ }^{20}=+30.1^{\circ}\left(c 0.41, \mathrm{CHCl}_{3}\right)\right)$ and $( \pm)-\mathbf{1 5}$ were isolated from Diplomorpha ganpi (Thymelaeaceae), $(-)-13\left([\alpha]_{\mathrm{D}}{ }^{20}=-31.4^{\circ}, c 0.74, \mathrm{CHCl}_{3}\right)$ was isolated from Diplomorpha canescens as a new compound ${ }^{29}$. Moreover, 4, 6 and (-)-14 were obtained from the stem barks of Wikstroemia coriacea (Thymelaeaceae) $^{30}$. Compound (-)-14, named as coriaceol was isolated from the stem bark of Wikstroemia coriacea. Although its exact stereochemistry could not be determined due to the decomposition at C-2, its specific optical rotation of $[\alpha]_{\mathrm{D}}^{25}=-12^{\circ}\left(c 0.02, \mathrm{CHCl}_{3}\right)$ might be attributed to be the enantiomer of $(+)-\mathbf{1 4}^{30}$. The known compounds 4, 5, 6 and (-)-erythro-20 were isolated from Diplomorpha sikokiana ${ }^{31}$.

$6\left([\alpha]_{\mathrm{D}}{ }^{18}=+4^{\circ}(c 0.275, \mathrm{MeOH}), S\right.$ configuration) from the roots of Stellera chamaejasme ${ }^{32,33,42}$ exhibited immunomodulatory and antitumor activities ${ }^{32}$ and toxic activity against Peries rapae $\mathbf{a}^{33} .6$ was also isolated from Euphorbia altotibetica (Euphorbiaceae) ${ }^{34}$.

A new diarylpentanoid 7, named kinsenone, having $(E, Z)$ configurations, from Anoectochilus formosanus (Orchidaceae), had strong antioxidant activity ${ }^{35}$.

Compound 8 which is a diastereomer of 7 , posessing $(E, E)$ configurations was purified from Curcuma longa. It was reported to have antiviral activity against influenza viruses ${ }^{36}$.

Natural products $\mathbf{9}$ and $\mathbf{1 0}$ were isolated from Daphne odora var. marginata. $\mathbf{1 0}$ was reported to exhibit cytotoxic activity against human tumor cell lines K562, A549, MCF-7, LOVO and HepG2 ${ }^{37}$. Compound 9 was also isolated from the root barks of Daphne giraldii ${ }^{38}$, which was given different names of daphneone and daphnolon.

Compounds 16 and 17, having specific optical rotations of $[\alpha]_{\mathrm{D}}{ }^{16}=-51.4^{\circ}(c 0.40, \mathrm{MeOH})(S)$ and $[\alpha] \mathrm{D}^{16}=-26.8^{\circ}(c 0.05, \mathrm{MeOH})(R)$, respectively, and $\mathbf{9}$ and 10, isolated from the roots and leaves of Daphne giraldii, were reported to display cytotoxic activities against human melanoma A375-S2 tumor cells. Their structures were elucidated using spectroscopic methods such as ${ }^{1} \mathrm{H}-\mathrm{NMR},{ }^{13} \mathrm{C}-\mathrm{NMR}$, twodimensional NMR, UV and $\mathrm{MS}^{39}$.

Compound 19 (Artamenone) was isolated from the stem and root barks of Artabotrys modestus ssp. macranthus (Annonaceae) ${ }^{40}$ and fruits of Lycium barbarum (Solanaceae) ${ }^{41}$.

Compound (-)-erythro-20 $\left([\alpha]_{\mathrm{D}}=-19.2(c 0.52, \mathrm{MeOH}) ; \mathrm{mp} .91-92{ }^{\circ} \mathrm{C}\right)$, a unique example of the natural 1,5-diarylpentanoids containing two $\mathrm{OH}$ groups in the $\mathrm{C} 5$ chain, was first isolated from the wood of Flindersia laevicarpa (Rutaceae) ${ }^{43}$. Later, (-)-erythro-20 was isolated from Wikstroemia sikokiana and its absolute configuration was established as $\mathbf{1}(S)$ and $\mathbf{3}(S)$ after a series of chemical synthesis ${ }^{44}$. 


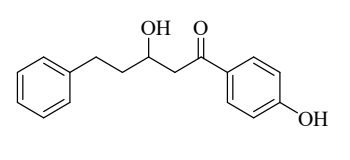

(+)-1

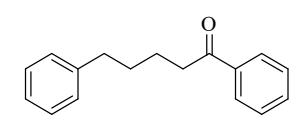

4
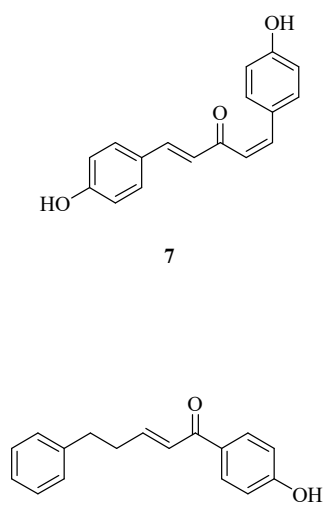

10

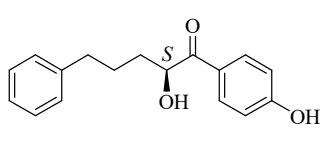

$(-)-13$

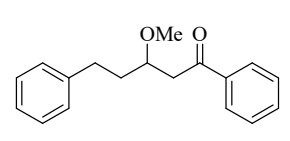

$( \pm)-15$

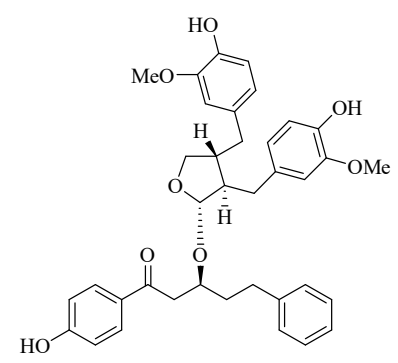

18

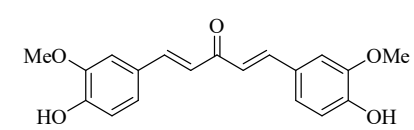

2

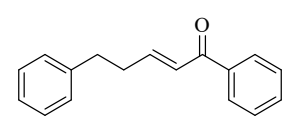

5
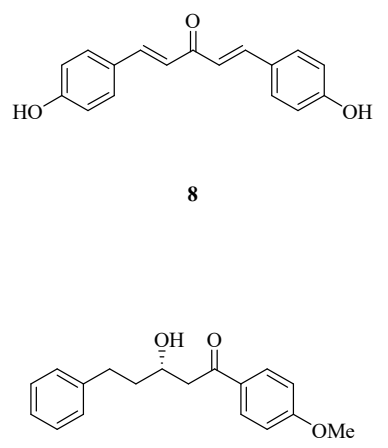

(+)-11

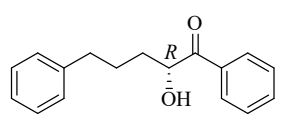

(+)-14

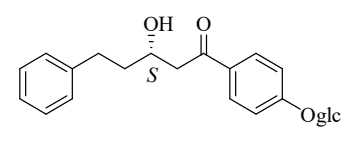

$(-)-16$

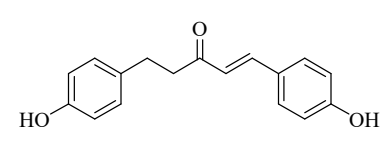

19

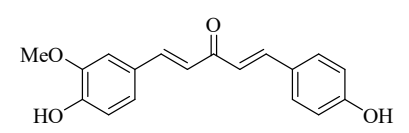

3

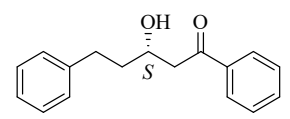

(+)-6

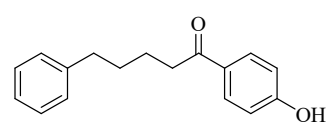

9

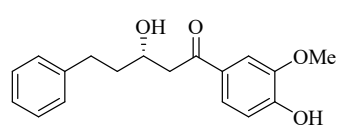

(+)-12

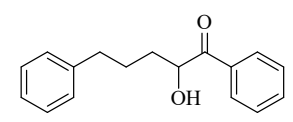

(-)-14

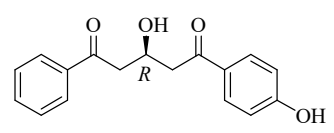

$(-)-17$

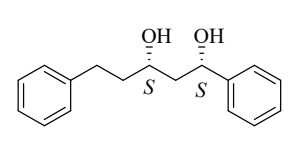

(-)-erythro-20

Figure 1. Diarylpentanoid compounds isolated from nature 
Table 1. Diarylpentanoids of structures, isolations and the reported biological activities

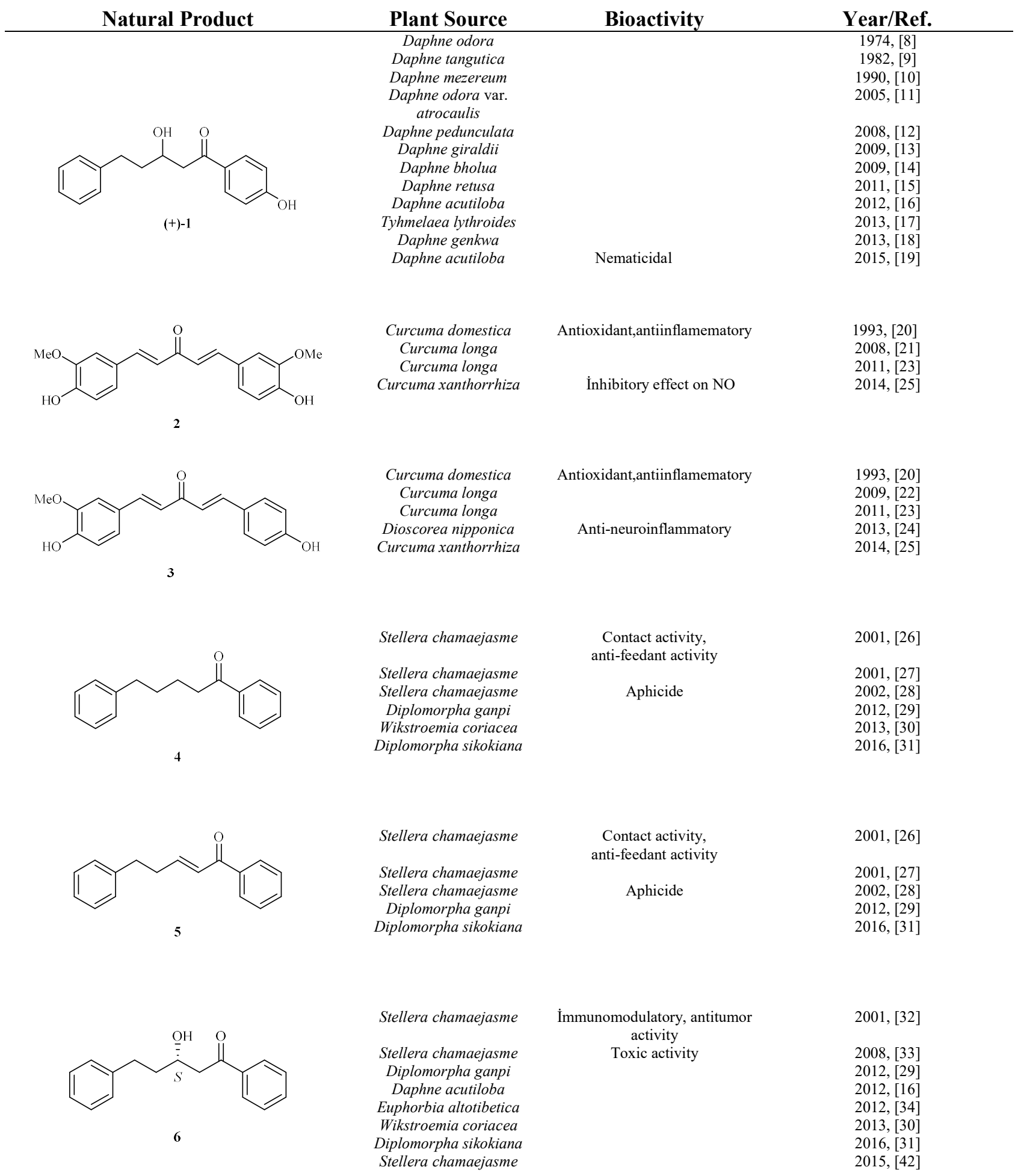


Natural Product<smiles>O=C(/C=C/c1ccc(O)cc1)/C=C/c1ccc(O)cc1</smiles><smiles>O=C(/C=C/c1ccc(O)cc1)/C=C/c1ccc(O)cc1</smiles>

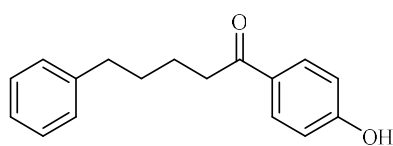

9

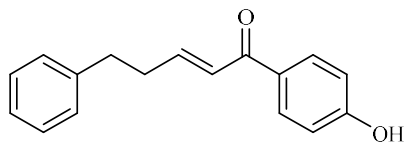

10<smiles>COc1ccc(C(=O)C[C@@H](O)CCc2ccccc2)cc1</smiles>

(+)-11<smiles>COc1cc(C(=O)C[C@@H](O)CCc2ccccc2)ccc1O</smiles>

$(+)-12$

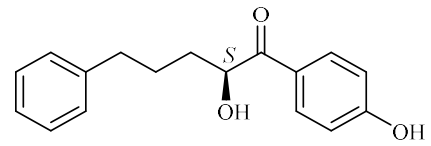

$(-)-13$
Plant Source

Bioactivity

Year/Ref.

Anoectochilus formosanus

Antioxidant

2002, [35]

Curcuma longa

Curcuma longa

Daphne odora var. marginata Daphne giraldii

Daphne giraldii

Daphne acutiloba

Tyhmelaea lythroides

Daphne acutiloba

Daphne odora

Daphne bholua

Daphne acutiloba

Daphne giraldii

Daphne genkwa

Daphne giraldii

Cytotoxicity

Nematicidal

Cytotoxicity

2006, [37]

2009, [14]

2012, [16]

Cytotoxicity

2012, [39]

2013, [18]

2009, [13]

Daphne giraldii

2009, [13]

Diplomorpha canescens

2012, [29] 
Natural Product<smiles>O=C(c1ccccc1)[C@@H](O)CCCc1ccccc1</smiles>

$(+)-14$<smiles>O=C(c1ccccc1)C(O)CCCc1ccccc1</smiles>

$(-)-14$<smiles>COC(CCc1ccccc1)CC(=O)c1ccccc1</smiles>

$( \pm)-15$<smiles>COc1ccc(C(=O)CC(O)CCc2ccccc2)cc1</smiles>

$(-)-16$<smiles>[X]C(O)CC(=O)c1ccc(O)cc1</smiles>

$(-)-17$<smiles>COc1cc(C[C@@H]2COC[C@@H]2Cc2ccc(O)c(OC)c2)ccc1O</smiles><smiles>COC(CCc1ccccc1)CC(=O)c1ccc(O)cc1</smiles>

18<smiles>O=C(/C=C/c1ccc(O)cc1)CCc1ccc(O)cc1</smiles>

19

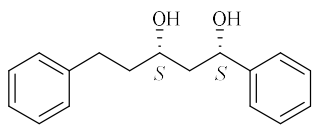

(-)- erythro-20
Plant Source

Bioactivity

Year/Ref.

Wikstroemia coriacea

Diplomorpha ganpi

Cytotoxicity

2012, [39]

Daphne giraldii

Cytotoxicity

2012, [39]

Daphne acutiloba

Anti-HIV

2012, [16]

Artabotrys modestus ssp.

macranthus

Lycium barbarum

2013, [40]

2014, [41]

Flindersia laevicarpa

Wikstroemia sikokiana

1962, [43]

Diplomorpha sikokiana 


\section{Conclusion}

This review surveyed the structures and biological activities of diarylpentanoids, which are rarely found compounds in nature. So far, only 20 natural diarylpentanoid compounds, having Ar-C5-Ar, were isolated.

In the light of phytochemical studies, we can say that diarylpentanoids have been found in the genus Daphne, Tyhmelaea, Curcuma, Stellera, Diplomorphai, Dioscorea, Wikstroemia, Anoectochilus, Euphorbia, Artabotrys and Lycium and the families Thymelaeaceae, Zingiberaceae, Dioscoreaceae, Euphorbiaceae, Orchidaceae, Annonaceae, Solanaceae, Rutaceeae.

We think that naturally occurring 1,5-diarylpentanoids can be models to develop biologically active drug-like compounds and further studies may discover new aspects of these compounds.

Acknowledgements: The reported rewiew is a part of $\mathrm{PhD}$ dissertation of Neslihan Celebioglu. We are thankful Dr. Necla Oztaskin and Umut Duaci for their kind help.

\section{ORCID}

Neslihan Celebioglu: 0000-0003-1882-0720

Ufuk Ozgen: 0000-0001-9839-6717

Hasan Secen: $\underline{0000-0002-5388-6111}$

\section{References}

[1] Claeson, P.; Tuchinda, P.; Reutrakul, V. Naturally occuring 1,7-Diarylheptanoids. J. Indian Chem. Soc. 1994, 71, 509-521.

[2] Claeson, P.; Claeson U. P.; Reutrakul, V. Occurrence structure and bioactivity of 1,7Diarylheptanoids. Stud. Nat. Prod. Chem. 2002, 26, 881-908.

[3] Lv, H.; She, G. Naturally occurring diarylheptanoids. Nat. Prod. Commun. 2010, 5, 1687-1708.

[4] Lv, H.; She, G. Naturally occurring diarylheptanoids-A Supplementary version. Rec. Nat. Prod. 2012, 6, 321-333.

[5] Cikrikci, S.; Mozioglu, E.; Yilmaz, H. Biological activity of curcuminoids isolated from Curcuma longa. Rec. Nat. Prod. 2008, 2, 19-24.

[6] Bener, M.; Ozyurek, M.; Guclu, K.; Apak, R. Optimization of microwave-assisted exraction of curcumin from Curcuma longa L. (turmeric) and evaluation of antioxidant activity in multi-test systems. Rec. Nat. Prod. 2016, 10, 542-554.

[7] Bar, F. M. A. Dihydropyridinone alkaloid artifacts from Curcuma longa and their anti-migration activity against HepG2 cells. Rec. Nat. Prod. 2016, 10, 582-589.

[8] Kogiso, S.; Hosozawa, S.; Wada, K.; Munakata, K. Daphneolone In roots of Daphne odora. Phytochemistry 1974, 13, 2332-2334.

[9] Lin-gen, Z.; Seligmann, O.; Jurcic, K.; Wagner, H. Constituents of Daphne tangutica. Planta Med. 1982, 45, 172-176.

[10] Kreher, B.; Neszmelyi, A.; Wagner, H. Triumbellin, a tricoumarin rhamnopyranoside from Daphne mezereum. Advances in the elegance of chemistry in designing dendrimers. Phytochemistry 1990, 29, 3633-3637.

[11] Zhang, W.; Zhang, W.; Li, T.; Liu, R.; Fu, P.; Li, H. Phenolic constituents from Daphne odora var. atrocaulis. Chanwu Yanjiu Yu Kaifa 2005, 17, 26-28; (Chem. Abstr. 2006, 146:138831).

[12] Xu, W.; Jin, H.; Zhang, W.; Fu, J.; Hu, X.; Zhang, W.; Yan, S.; Shen. Y. Studies on the chemical constituents of Daphne pedunculata. Chem. Nat. Compd. 2008, 44, 771-772.

[13] Wu, H. Z.; Wang, B. L.; Gao, Y. H.; Huang, J.; Sun, H. B.; Li, H. S.; Wu. J. L. The chemical constituents of the tissue culture cells of Daphne giraldii cullus. Chin. Chem. Lett. 2009, 20, 13351338.

[14] Chen, H.; Zhang, W.; Su, J.; Chen. Y.; Shen, Y. Phenols constituents in stem and leaf of Daphne bholua. Zhongcaoyao 2009, 40, 1033-1035; (Chem. Abstr. 2010, 154:405145). 
[15] Hu, X.; Jin, H.; Yan, L.; Zhang, W. Chemical constituents of Daphne retusa. Tianran Chanwu Yanjiu Yu Kaifa 2011, 23, 20-24; (Chem. Abstr. 2011, 156:431236).

[16] Huang, Z. S.; Zhang, J. X.; Li, Y. X.; Jiang, Z. H.; Ma, Y. Q.; Wang, C. P.; Liu, Q. Y.; Hu, M. J.; Zheng, T. Y.; Zhou, J.; Zhao, X. Y. Phenols with anti-HIV activity from Daphne acutiloba. Planta Med. 2012, 78, 182-185.

[17] Kabbaj, Z. F.; Lai, D.; Meddah, B.; Altenbach, HJ.; Cherrah, Y.; Proksch, P.; Faouzi, A. E. M.; Debbab, A. Chemical constituents from aerial parts of Thymelaea lythroides. Biochem. Syst. Ecol. 2013, 51, 153-155.

[18] Chen, YY.; Duan, JA.; Tang, YP.; Guo, S. Chemical constituents from flower buds of Daphne genkwa. Zhongcaoyao 2013, 44, 397-402; (Chem. Abstr. 2013, 160:317277).

[19] Zhuo, S. H.; Ni, H. H.; Yun, Q. M.; He, M. M. Liang, M. Z.; Fu, H. D.; Ping, Y. J.; Hai, Q. W.; Xing, Y.Z. The phytochemicals with antagonistic activities toward pathogens of a disease complex caused by Meloidogyne incognita and Ralstonia solanacearum. J. Pure. Appl. Microbiol. 2015, 9, 209-213.

[20] Masuda, T.; Jitoe, A.; Isobe, J.; Nakatani, N.; Yonemori, S. Anti-Oxidative and Anti-Inflammatory curcumin-related phenolics from rhizomes of Curcuma domestica. Phytochemistry 1993, 32, 15571560 .

[21] Wang, LY.; Zhang, M.; Zhang, CF.; Wang, ZT. Diaryl derivatives from the root tuber of Curcuma longa. Biochem. Syst. Ecol. 2008, 36, 476-480.

[22] Li, W.; Wang, S.; Feng, J.; Xiao Y.; Xue, X.; Zhang, H.; Wang, Y.; Liang, X. Structure elucidation and NMR assignments for curcuminoids from the rhizomes of Curcuma longa. Magn. Reson. Chem. 2009, 47, 902-908.

[23] Xiao, C. Y.; Xie, J.; Yu, M.; Liu, M.; Ran, J.; Xi, Z.; Li, W.; Huang, J. Bisabocurcumin, a new skeleton curcuminoid from the rhizomes of Curcuma longa L. Chin. Chem. Lett. 2011, 22, 14571460 .

[24] Woo, W. K.; Moon, E.; Kwon, W. O.; Lee, O. S.; Kim, Y. S.; Choi, Z. S.; Son, W. M.; Lee, R. K.. Anti-neuroinflammatory diarylheptanoids from the rhizomes of Dioscorea nipponica. Bioorg. Med. Chem. Lett. 2013, 23, 3806-3809.

[25] Park, JH.; Jung, YJ.; Shrestha, S.; Lee, M. S.; Lee, H. T.; Lee, CH.; Han, D.; Kim, J.; Baek, NI. Inhibition of NO Production in LPS-Stimulated RAW264.7 Macrophage cells with curcuminoids and xanthorrhizol from the rhizome of Curcuma xanthorrhiza Roxb. and quantitative analysis using HPLC. J. Korean Soc. Appl. Biol. Chem. 2014, 57, 407-412.

[26] Ping, G.; Taiping, H.; Rong, G.; Qiu, C.; Shigui, L. Activity of the botanical aphicides 1,5diphenyl-1-pentanone and 1,5-diphenyl-2-penten-1-one on two species of Aphididnae. Pest. Manag. Sci. 2001, 57, 307-310.

[27] Feng, B.; Pei, Y.; Hua, H. Structure determination of constituents from Stellera chamaejasme. Zhongguo Yaowu Huaxue Zazhi 2001, 11, 112-114; (Chem. Abstr. 2001, 136:147802).

[28] Hou, TP.; Ciu, Q.; Chen, SH.; Hou, RT.; Liu, SG. New compounds against aphides from Stellera chamaejasme L. Youji Huaxue 2002, 22, 67-70; (Chem. Abstr. 2002, 136:180679).

[29] Devkota, P. H.; Watanabe, M.; Watanabe, T.; Yahara, S.; Diarylpentanoids from Diplomorpha canescens and Diplomorpha ganpi. Phytochem. Lett. 2012, 5, 284-286.

[30] Ingert, N.; Bombarda, I.; Herbette, G.; Faure, R.; Moretti, C.; Raharivelomanana, P. Oleodaphnoic acid and coriaceol, two new natural products from the stem bark of Wikstroemia coriacea. Molecules 2013, 18, 2988-2996.

[31] Devkota, P. H.; Joshi, R. K.; Watanabe, T.; Yahara, S. Chemical constituents from the roots, stems, and leaves of Diplomorpha sikokiana. Nat. Prod. Commun. 2016, 11, 475-476.

[32] Xu, ZH.; Qin, GW.; Xu, RS. A new bicoumarin from Stellera chamaejasme L. J. Asian Nat. Prod. Res. 2001, 3, 335-340.

[33] Liu, Q.; Jia, H.; Xiao, B.; Chen, L.; Zhou, B.; Hou, T. P. A new compound against Peries rapae from Stellera chamaejasme. Nat. Prod. Res. 2008, 22, 348-352.

[34] Yang, AM.; Liu, JL.; Sun, J.; Han, H.; Li, H.; Shi, XL. Chemical constituents from Euphorbia altotibetica. Zhongyiyao Xuebao 2012, 40, 71-73; (Chem. Abstr. 2012, 157:571287).

[35] Wang, SY.; Kuo, YH.; Chang, HN.; Kang, PL.; Tsay, HS.; Lin, KF.; Yang, NS.; Shyur, LF. Profiling and characterization antioxidant activities in Anoectochilus formosanus Hayata. J. Agric. Food. Chem. 2002, 50, 1859-1865. 
[36] Dao, T. T.; Nguyen, H. P.; Won, K. H.; Kim, H. E.; Park, J.; Won, Y. B.; Oh, K. W. Curcuminoids from Curcuma longa and their inhibitory activities on influenza A neuraminidases. Food Chem. 2012, 134, 21-28.

[37] Zhang, W.; Zhang, D. W.; Liu, H. R.; Shen, H, Y.; Zhang, C.; Cheng, S. H.; Fu, P.; Shan, L. Two new chemical constituents from Daphne odora Thunb. var. marginata. Nat. Prod. Res. 2006, 20, 1290-1294.

[38] Sun, WX.; Zhang, Q.; Jiang, JQ. Chemical constituents of Daphne giraldii Nitsche. J. Integrative Plant Biol. 2006, 48, 1498-1501.

[39] Wang, LB.; Dong, NW.; Wu, ZH.; Wu, LJ. Two new compounds with cytotoxic activity on the human melanoma A375-S2 cells from Daphne giraldii callus cells. J. Asian Nat. Prod. Res. 2012, 14, 1020-1026.

[40] Nyandoro, S. S.; Joseph, C. C.; Nkunya, H. H. M.; Hosea, M. M. K. New antimicrobial, mosquito larvicidal and other metabolites from two Artabotrys species. Nat. Prod. Res. 2013, 27, 1450-1458.

[41] Gao, K.; Tang, HF.; Lu, YY.; Wang, XY.; Zhang, W.; Ma, N. Chemical constituents in fruits of Lycium barbarum L. Zhongnan Yaoxue 2014, 12, 324-327; (Chem. Abstr. 2014, 162:143724).

[42] Chen, W.; Luo, XH.; Wang, Z.; Zhang, YY.; Liu, LP.; Wang, HB. A new biflavone glucoside from the roots of Stellera chamaejasme. Chin. J. Nat. Med. 2015, 13, 550-553.

[43] Breen, G.J.W.; Ritchie, E.; Taylor, W.C. The chemical constituents of australian flindersia species. XVI. The constutients of the wood of Flindersia laevicarpa C.T. White and Francis. Aust. J. Chem. 1962, 15, 819-823.

[44] Niwa, M.; Jiang, PF.; Hirata, Y. Constituents of Wikstroemia sikokiana II Absolute configurations of 1,5-diphenylpentane-1,3-diols. Chem. Pharm. Bull. 1987, 35, 108-111.

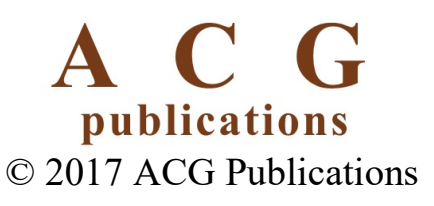

